\title{
Ophthalmological features of Aicardi's syndrome: report of two cases
}

\author{
E. E. LIMNAIOS, C. P. PANAYIOTOPOULOS, G. THEODOSIADIS, \\ M. ECONOMOU, AND T. PAPAPETROPOULOS
}

From the Department of Neurology, Division of Neuro-ophthalmology, University of Athens, Athens, Greece

SUMmaRY Two Greek female patients with Aicardi's syndrome are presented. The clinical picture, electroencephalogram, computer axial tomography scan, and the ophthalmological findings in these patients are described. The ocular features are emphasised because they can establish the diagnosis of this rare syndrome.

In 1965 Aicardi, Lefebvre, and Lerique-Koechlin described in a brief report a new syndrome consisting mainly of spasms in flexion, agenesis of the corpus callosum, and ocular anomalies (Aicardi et al., 1965). This clinical entity is now known as Aicardi's syndrome and from various reports it seems to have the following characteristics. Clinical manifestations include infantile spasms. Only females are attacked. Mental retardation occurs. Pneumoencephalography or computer axial tomography (CT-scan) shows agenesis of the corpus callosum. The EEG shows hypsarrhythmia and asynchronous activity of the 2 hemispheres (Fariello et al., 1977). There are skeletal anomalies such as scoliosis, fusion of the vertebral bodies, and hemivertebrae. Ocular features include choroidoretinal 'lacunae' and more rarely colobomas of the optic disc, persistent pupillary membrane, staphylomas, proliferation of glial tissue at the optic disc, and microphthalmia.

Histopathological studies have shown absence of the pineal gland in addition to agenesis of the corpus callosum (de Jong et al., 1976). As regards the intraocular anomalies, the main interest has been focused on the chorioretinal lacunae. They have been found after fluorescein angiography as fluorescein-negative areas, suggesting choroidal atrophy (Hammami et al., 1972). Detailed histological examination has shown that at the lacunae the retina is normal, the pigment epithelium is depigmented but intact, and the choroid has gross atrophy (Bary, 1973; de Jong et al., 1976).

The ocular and central nervous system abnormalities are thought to occur between the 4th and

Correspondence to Dr E. E. Limnaios, Aiginition Hospital, Athens, Greece. 7th week of intrauterine life. The retinal pigment epithelium appears in the 4th week, the choroidal pigment appears in the 5th week, and the commissural plate is formed from the 4th to the 7th week, although the full development of the corpus callosum takes up to the 3rd month of fetal life to be completed (Andreu, 1973). The various defects we often see in this area can be explained by the long time taken for the midline structures of the brain to be formed (Saraux et al., 1969).

The absolute restriction of the syndrome to the female sex was by Aicardi et al. (1965) to the presence of a single dominant gene on the $\mathrm{X}$ chromosome, which is produced by a spontaneous mutation and is lethal to the male. On the other hand de Jong et al. (1976), after reporting no evidence of pineal gland in 2 patients with Aicardi's syndrome and regarding this gland as an important biological clock for sexual development, believe that the absence of the gland is incompatible with the development of a viable male child regardless of the cause of the syndrome.

The prime criteria for the diagnosis of Aicardi's syndrome are infantile spasms, agenesis of the corpus callosum, and chorioretinal 'lacunae.' With these criteria some 78 cases of the syndrome in children have been reported from various parts of the world. We report on the first 2 cases found in Greece.

\section{Case reports}

CASE 1

The patient was a girl aged 25 months. She was born at term to 2 healthy parents; the mother was 30 and the father was 35 years old. There was also a healthy son of 9 years in the family. No family history of ocular or neurological disorders was reported. 
The patient did well until the 4th month of her life. Then she began to have tonic spasms of the trunk and both upper and lower limbs, with synchronous turning of the head and eyes to the left. Each episode lasted for a few seconds. The frequency of the attacks was 3 to 5 per day.

On examination she had a right hemiparesis. She responded well to photic stimuli. Ocular motility and optokinetic responses were normal. A rough evaluation of visual fields by using familiar toys and lights of various colours showed no gross field defects. The pupillary reaction to light and near vision was also normal. Examination of the anterior segment gave no abnormal findings. In the right fundus the optic disc was normal, with an excess of

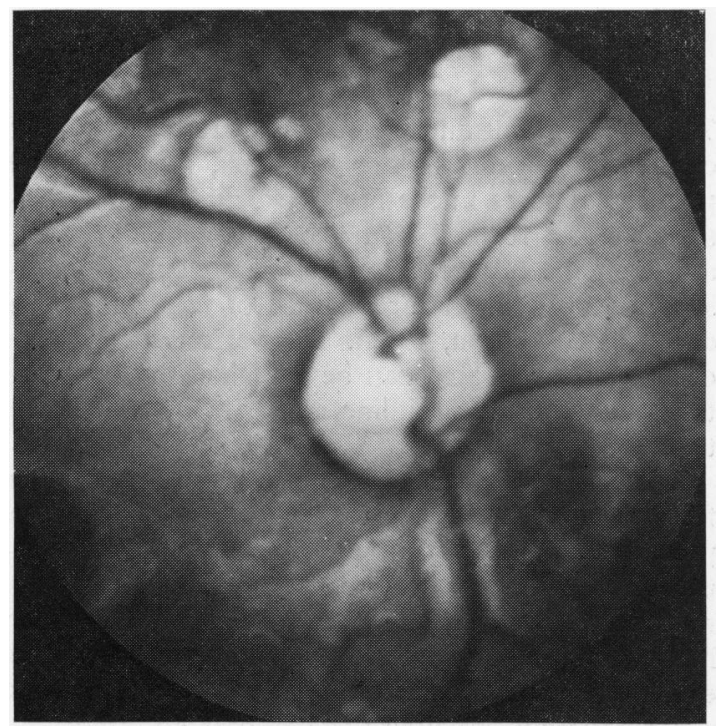

Fig. 1 Case 1. Large chorioretinal 'lacunae' in the right fundus

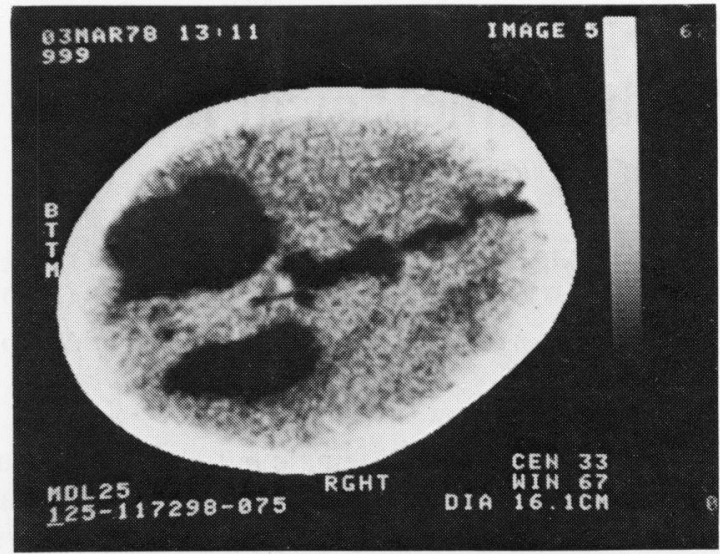

Fig. 3 Case 1. CT-scan shows agenesis of the corpus callosum and ectopic parenchyma in the left lateral ventricle

black pigment round it. Close to the optic disc and at the 1 and 11 o'clock positions we found 2 well defined chorioretinal lesions of white-yellow colour with some pigment at their border. Below the optic disc and at the 4 o'clock position an area of deeper pigmentation was seen. The blood vessels passed over the lesions without interruption (Fig. 1). In the left fundus the optic disc was also normal. Pale yellow lesions of less than $0 \cdot 1$ disc diameter were seen round the posterior pole.

The EEG showed hypsarrhythmia, which was markedly asynchronous and more pronounced in the left hemisphere (Fig. 2). The CT-scan showed agenesis of the corpus callosum and ectopic parenchyma in the left lateral ventricle (Fig. 3). Laboratory tests for venereal disease and Reiter's syndrome were all negative.

The patient was treated with ACTH, nitrazepam, phenobarbitone, and sodium valproate. The EEG
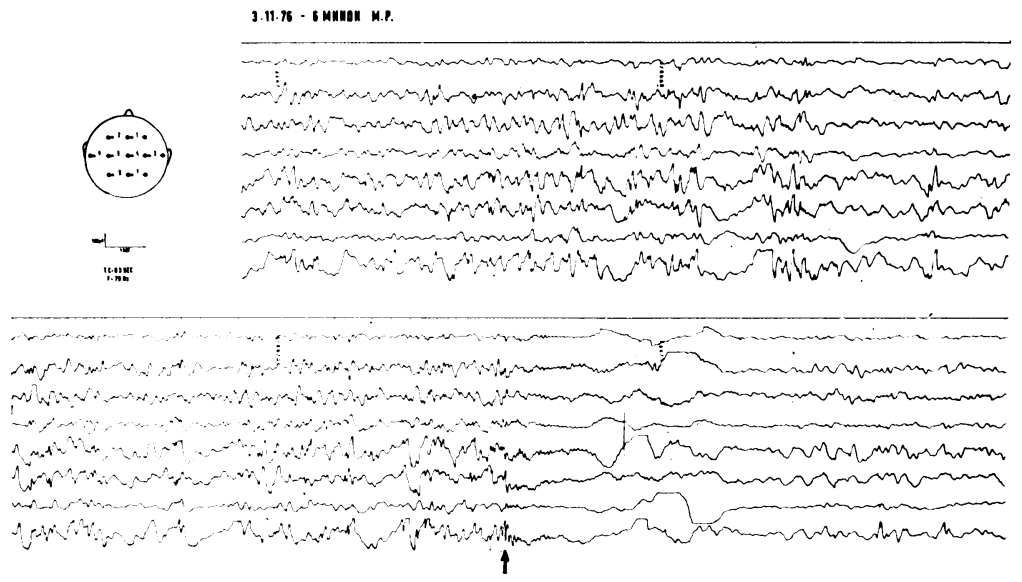

Fig. 2 Case 1. EEG recorded at the age of 6 months. Gross abnormalities mainly on the left. At the arrow the patient had a fiexion spasm 
improved and the epileptic fits were reduced to 1 per week. She had still a moderate right hemiparesis and developed mild mental retardation.

\section{CASE 2}

The patient was a girl 6 months old. She was the first and only child of healthy parents, the mother being aged 32 and the father 36 . She was born at term after a normal pregnancy. There was no family history of ocular or neurological disease.

In the fourth month of her life she began to have tonic spasms of the upper and lower limbs, flexing them and turning the head and eyes to the right. An

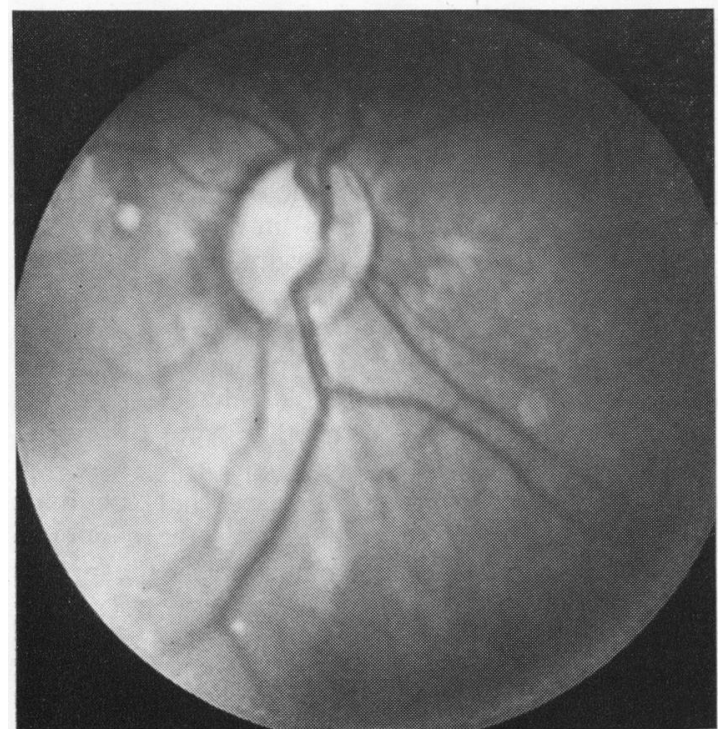

Fig. 4 Case 2. Small chorioretinal lesions in the right fundus

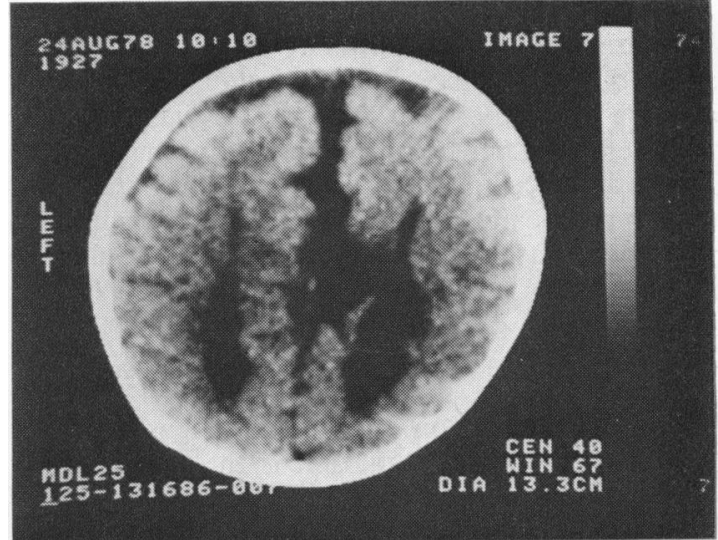

Fig. 6 Case 2. CT-scan shows agenesis of the corpus callosum

estimated number of 50 spasms were seen on each attack, and the whole episode lasted for a few seconds, to be repeated about 8 times every day.

On examination she was found to have a right hemiparesis. She responded well to photic stimuli. The ocular motility and optokinetic responses were normal. The pupils were equal and responded well to light. The structures of the anterior segment were normal and the vitreous was clear. The optic discs and the central retinal vessels were also normal. In the posterior pole of both eyes small, round, pale yellow lesions were seen, of less than $0 \cdot 1$ disc diameter. Deeper pigmentation was seen at the border of each lesion (Fig. 4).

The EEG showed hypsarrhythmia and was markedly asynchronous (Fig. 5). The CT-scan was typical of agenesis of the corpus callosum (Fig. 6). Laboratory investigations were negative.
Fig. 5 Case 2. EEG recorded at the age of 6 months. Upper: Awake EEG. Lower: Sleep EEG. Gross abnormalities mainly on the right
9.10.78 - 7 MHNON- 8.T.
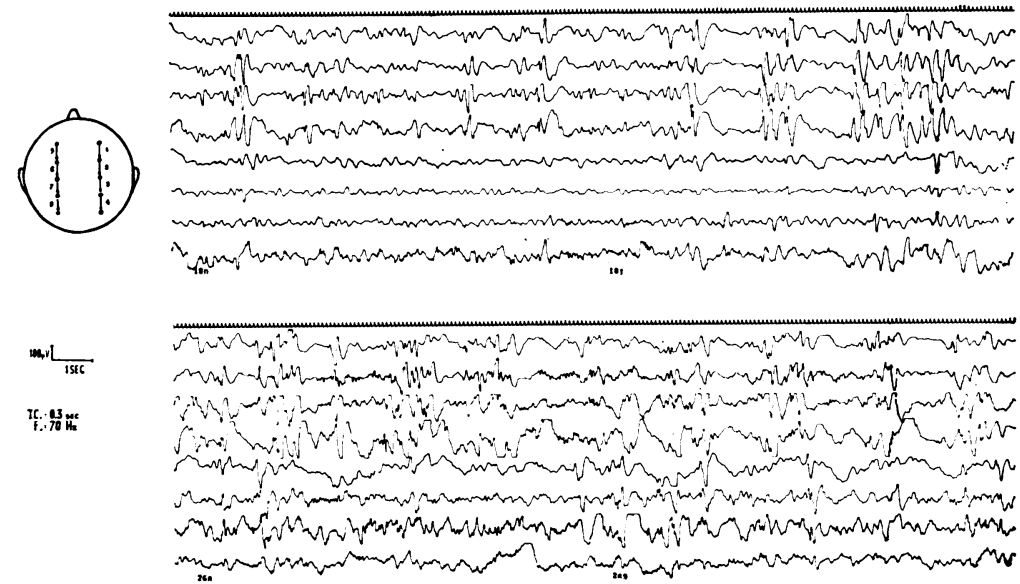
The patient was treated with ACTH, phenobarbitone, and clonazepam. The spasms then stopped, the hemiparesis cleared almost completely, and the EEG improved. The ACTH was gradually removed from her treatment. Two months after the initial attack she was taking phenobarbitone and clonazepam, and the only remaining symptoms were mild spasms of short duration in the right arm twice a week.

\section{Discussion}

The proper evaluation of the ocular signs in female infants with spasms in flexion is the responsibility of the clinical ophthalmologist, who can exclude or establish the diagnosis of Aicardi's syndrome. Each patient may present a different ophthalmoscopic picture, but there are points in common suggesting the diagnosis of the syndrome.

The examination for the basic visual function of our patients showed normal responses to photic stimuli and normal fixation and following movements. In other reports the eyes have been found to show more pronounced anomalies and impaired visual function (Aicardi and Chevrie, 1969; Rousselie and Fontaine, 1971). The pupils in our patients were normal in size, shape, and reaction. Other reported cases have shown persistent pupillary membrane, anterior or posterior synechiae, and an abnormal or absent pupillary reaction to light (Aicardi et al., 1969; Hoyt et al., 1978). Strabismus has been reported in some cases (Rousselie and Fontaine, 1971), but in our patients we found no strabismus or abnormal ocular motility. Although microphthalmos or exophthalmos are reported by Aicardi et al. (1969), the appearance of the eyes in our patients was normal.

Hoyt et al. (1978), discussing the possibility of an infectious agent contributing to the pathogenesis of Aicardi's syndrome, refer to the observation of an inflammatory reaction in the anterior vitreous in one of their patients. The clear vitreous in both eyes of our patients does not support this view.

The most interesting ophthalmological findings in Aicardi's syndrome are those of the fundus. The optic disc in our patients was normal in both eyes. Excessive pigmentation in the peripapillary area was observed in the eye with the more severe chorioretinal lesions. Colobomas of the optic disc, reported fairly often, were not seen in our cases. Aicardi et al. (1969) have found 4 colobomas of the optic disc in 15 patients and Hoyt et al. (1978) 3 in 4 patients. Moreover, preretinal masses of glial tissue noted in other reports (for example, Rousselie and Fontaine, 1971) were not observed in our cases. The retinal vessels in the 4 eyes we have examined were normal. The haemorrhages in the retina reported by Deodati et al. (1974) were obviously a transient phenomenon.

The pathognomonic ocular feature of Aicardi's syndrome is the chorioretinal 'lacuna.' Aicardie (1965) in his original report describes the lacunae as areas of white-yellow colour ranging in size from $0 \cdot 1$ disc diameter to twice that of the normal disc. In our own cases lesions were found in both eyes. Comparing these lesions with those seen in fundus photographs of similar reports, we conclude that there are 2 main types of chorioretinal lesions. The first is the large and well defined hypopigmented lacuna with irregular pigment clumping at its border. Two such large lesions were found in the right eye of our case 1 . The second is the small yellow lesion found in both eyes of our patients. It is important at this point to emphasise that the small lesions are as pathognomonic as the large ones for the diagnosis of Aicardi's syndrome when the other 2 criteria are present, namely, the spasms in flexion and the agenesis of the corpus callosum. An additional diagnostic feature is the small amount of pigment which surrounds large and small lesions and is in contrast with their distinctly hypopigmented central area (Aicardi et al., 1965). This can be taken as a point of differentiation from other types of chorioretinal lesions.

We conclude from our 2 cases that the main assistance the clinical ophthalmologist can offer in the diagnosis of Aicardi's syndrome is the proper evaluation of the chorioretinal lesions.

\section{References}

Aicardi, J., Lefebvre, J., and Lerique-Koechlin, A. (1965). A new syndrome: spasm in flexion, callosal agenesis, ocular abnormalities. Electroencephalography and Clinical Neurophysiology, 19, 609-610.

Aicardi, J., and Chevrie, J. J. (1969). Le syndrome agénésie calleuse, spasmes en flexion, lacunes chorio-rétiniennes. Archives Françaises de Pédiatrie, 26, 809-810.

Aicardie, J., Chevrie, J. J., and Rousselie, F. (1969). Le syndrome spasme en flexion, agénésie calleuse, anomalies chorio-rétiniennes. Archives Françaises de Pédiatrie, 26, 1103-1120.

Andreu, A. (1973). Le syndrome d'Aicardi, Thèse, Université Paul-Sabatier, Toulouse.

Bary, A. (1973). Aicardi's syndrome. Meeting of the European Ophthalmic Pathology Society, May 17-19.

de Jong, J. G. Y., Delleman, J. W., Houben, M., Manschot, W. A., de Minjer, A., Mol, J., and Slooff, J. L. (1976). Agenesis of the corpus callosum, infantile spasms, ocular anomalies (Aicardi's syndrome). Neurology, 26, 11521158.

Deodati, F., Bec, P., Carriere, J. P., and Labro, J. B. (1974). Le syndrome d'Aicardi. Archives d'Ophthalmologie et Revue Générale d'Ophthalmologie, 34, 85-98.

Fariello, R. G., Chun, R. W. M., Doro, J. M., Buncic, J. R., and Prichard, J. S. (1977). EEG recognition of Aicardi's syndrome. Archives of Neurology, 34, 563-568. 
Hammami, H., Streiff, E. B., and de Wolff, E. (1972). Contribution à la connaissance du syndrome d'Aicardi et coll. Bulletins et Mémoires de la Société Française d'Ophthalmologie, 85, 598-614.

Hoyt, C. S., Billson, F., Ouvrier, R., and Wise, G. (1978). Ocular features of Aicardi's syndrome. Archives of Ophthalmology, 96, 291-295.
Rousselie, F., and Fontaine, M. (1971). Anomalies choriorétiniennes. Spasmes en flexion. Agénésie calleuse. Archives d'Ophthalmologie et revue Générale d'Ophthalmologie, 31, 539-546.

Saraux, H., Biais, B., and Chatellier, P. (1969). Anomalies oculaires et malformations de structures medianes du cerveau antérieur. Annales d'Oculistique, 202, 241-257. 